\title{
Protein synthesis and degradation are required for the incorporation of modified information into the pre-existing object-location memory
}

\author{
Jun-Hyeok Choi ${ }^{1}$, Jung-Eun Kim¹, Bong-Kiun Kaang ${ }^{1,2^{*}}$
}

\begin{abstract}
Although some reports indicate that protein synthesis dependent process may be induced by updating information, the role of protein synthesis and degradation in changing the content of pre-existing memory is yet unclear. In this study, we utilized an object rearrangement task, in which partial information related to a pre-existing memory is changed, promoting memory modification. Inhibitors of both protein synthesis and protein degradation impaired adequate incorporation of the altered information, each in a distinctive way. These results indicate that protein synthesis and degradation play key roles in memory modification.
\end{abstract}

\section{Introduction}

Memory formation in real life usually involves partial change of pre-existing memory. Though numerous studies have highlighted the molecular mechanism of memory formation, it is largely unknown how previously formed memory is altered or updated in the process.

A candidate mechanism of memory updating could involve dynamic regulation of memory stability after memory reactivation. Conventionally, it was thought that memory is consolidated by a protein synthesisdependent process only once per item and persists thereafter in a stable state. However, accumulating evidence suggests that memory reactivation induces a reconsolidating process that depends on the protein synthesis [1-4]. While protein synthesis has been shown to be required for the memory restabilization after retrieval, the destabilizing phase seems to require protein degradation. These studies showed that, using the contextual fear memory task, protein degradation in the hippocampus after memory reactivation is required for weakening or strengthening of the fear memory $[5,6]$. This dynamic protein turnover after memory reactivation is hypothesized to be a molecular mechanism through which memory is updated or modified. Indeed, several reports have suggested that this protein-

\footnotetext{
* Correspondence: Kaang@snu.ac.kr

${ }^{1}$ National Creative Research Initiative Center for Memory, Department of Biological Sciences, College of Natural Sciences, Seoul National University,
} 151-742 Seoul, Korea

(c) 2010 Choi et al; licensee BioMed Central Ltd. This is an Open Access article distributed under the terms of the Creative Commons Attribution License (http://creativecommons.org/licenses/by/2.0), which permits unrestricted use, distribution, and reproduction in any medium, provided the original work is properly cited.

To assess the incorporation of changed information into the preexisting memory, we chose an object-location memory task [11]. This task measures object-location associative memory utilizing the innate tendency of rodents to explore the novel aspects of the environment. The scheme of the task is depicted in Figure 1A. Nineto 11 -week-old $\mathrm{C} 57 \mathrm{BL} / 6 \mathrm{~N}$ male mice were used for all the experiments, housed as described previously [12]. After five days of 15 minutes habituation to a context, the mouse was exposed for 15 minutes to four objects located in one of the four positions in the context. Twenty-four hours later, the mouse was re-exposed to

synthesis-inhibitor-sensitive reconsolidation process folexternal stimulus that promotes updating of the original information [7-10].

However, the previous studies only focused on the ment of protein synthesis and degradation in the incorporation of changed information. Another major memory updating process in real-life is partially modifying the content of the initial memory rather than simply strengthening or weakening the memory. In the present
study, we aimed to reveal the role of protein synthesis and degradation in the incorporation of partially modified information into the pre-existing memory, by using an object rearrangement task.

C Biomed Central medium, provided the original work is properly cited. 
the context for 15 minutes with the location of two adjacent objects inter-switched while the other two left unchanged. Higher explorative preference to the switched objects was expected due to the novelty of the situation. On the next day, the mouse was re-exposed for 15 minutes to the same object configuration as the second day. If it had successfully incorporated the changed information on the second day, there would be no novel aspects in each object anymore, resulting in similar preference for each of them.

To evaluate the preference for switched/unswitched objects, we measured the duration of exploration time for each object and calculated the percentage of the two switched/unswitched objects exploration time from the total exploration time. The preference to the switched objects was higher on day 2 , indicating that the subjects have well recognized the original position or configuration of each object (Figure 1B). This returned to chance level at day 3 when the same configuration as day 2 was given. This implies that the subjects have well memorized the new, changed configuration to recognize it as familiar.

Although there was a slight difference in the preference for each of the four objects at the first day, it was statistically non-significant (Figure 1C). The two objects with slightly higher preference were always paired with the objects with lower preference, and the pair itself was counterbalanced for the next experiment.

As our aim was to focus on the associative memory between the objects and their location, we targeted hippocampus which seems to be more specifically involved in object-location memory $[13,14]$. Hippocampus is known to be important for object-location memory [14-17]; although other regions such as MPFC and perirhinal cortex are also implicated in such tasks, they are probably more involved in novelty processing. Especially, it was recently reported that PKMzeta inhibitor destructed object location memory, but not object identity memory, when it was locally applied in hippocampus [18]. We implanted guide cannulae one week before the behavioral procedure and injected the protein synthesis inhibitor anisomycin (Ani; $200 \mu \mathrm{g} / \mu \mathrm{l}$ in aCSF, $0.3 \mu \mathrm{l}$ ) and/or the proteasome inhibitor clasto-lactacystin- $\beta$-lactone ( $\beta$ lac; $32 \mathrm{ng} / \mu \mathrm{l}$ in aCSF, $0.3 \mu \mathrm{l}$ ) or vehicle (Veh; aCSF, $0.3 \mu \mathrm{l}$ ) in the CA1 region of dorsal hippocampus, bilaterally, right after the exposure to the changed object location on day 2 (Figure 2A). Position of the cannulae tips are shown in Figure 2B.

On day 3, the anisomycin-injected group showed higher preference to the switched object compared to the vehicle-injected control group (Figure $2 \mathrm{C}$ ), suggesting that subjects could not incorporate the information from the modified object configuration on day 2. On the other hand, the $\beta$ lac-injected group showed higher preference to the unswitched object compared to vehicle control group. This suggests that the preexisting information (unchanged configuration) was relatively weakened or somewhat impaired compared to the new information (changed configuration). This is consistent with the findings that $\beta$ lac interrupted the reconsolidation process (Lee et al, 2008). It is still possible that the new information was more effectively stored in the presence of $\beta$ lac, thus increasing the relative familiarity with the switched objects over with the unswitched objects. It is noteworthy that the effects of anisomycin and $\beta$ lac on the memory change were opposite [preference to the switched objects (anisomycin) vs. to the unswitched ( $\beta$ lac)] as are the opposite effects of these drugs on protein level]. When anisomycin and $\beta$ lac were injected together, the preference was similar to the control group. The treatment of anisomycin and $\beta$ lac at the same time offset their effects on the behavioral change. The preference for switched object, on day 2 was similar among the four groups (Figure 2D). Taken together, our data suggest that protein synthesis and degradation have important roles in regulating the process of modifying the previously formed memory.

\section{Discussion}

In this study, we have provided a novel scheme of object-in-place memory task to assess whether the mice have correctly changed the contents of pre-existing object-location associative memory, making it possible to investigate the process of modifying memory. In previous studies focusing on the modification of pre-formed memory, the memory task commonly involved strengthening the memory by repeated training over days or weakening it by an experimental extinction protocol. Our modified object rearrangement task has an advantage that the memory is not simply altered in its strength, but that the partial contents of the pre-formed memory are rapidly reorganized to incorporate changed information. Based on this advantage, this task is likely to be valuable for studies on dynamic memory modification process. Using this task, we have found that protein synthesis and degradation are required for incorporating partially modified information into the pre-existing memory.

Considering the effect of anisomycin on the memory modification, the original memory might be expected to be destabilized by anisomycin after reactivation on day 2 as it would undergo a protein synthesis dependent reconsolidation process, possibly resulting in the chance level preference for each object on day 3 . However, anisomycin-injected mice acted on day 3 as if the second object configuration is novel, resulting in higher preference for the switched objects. One possible explanation for this is that not every component of the related 


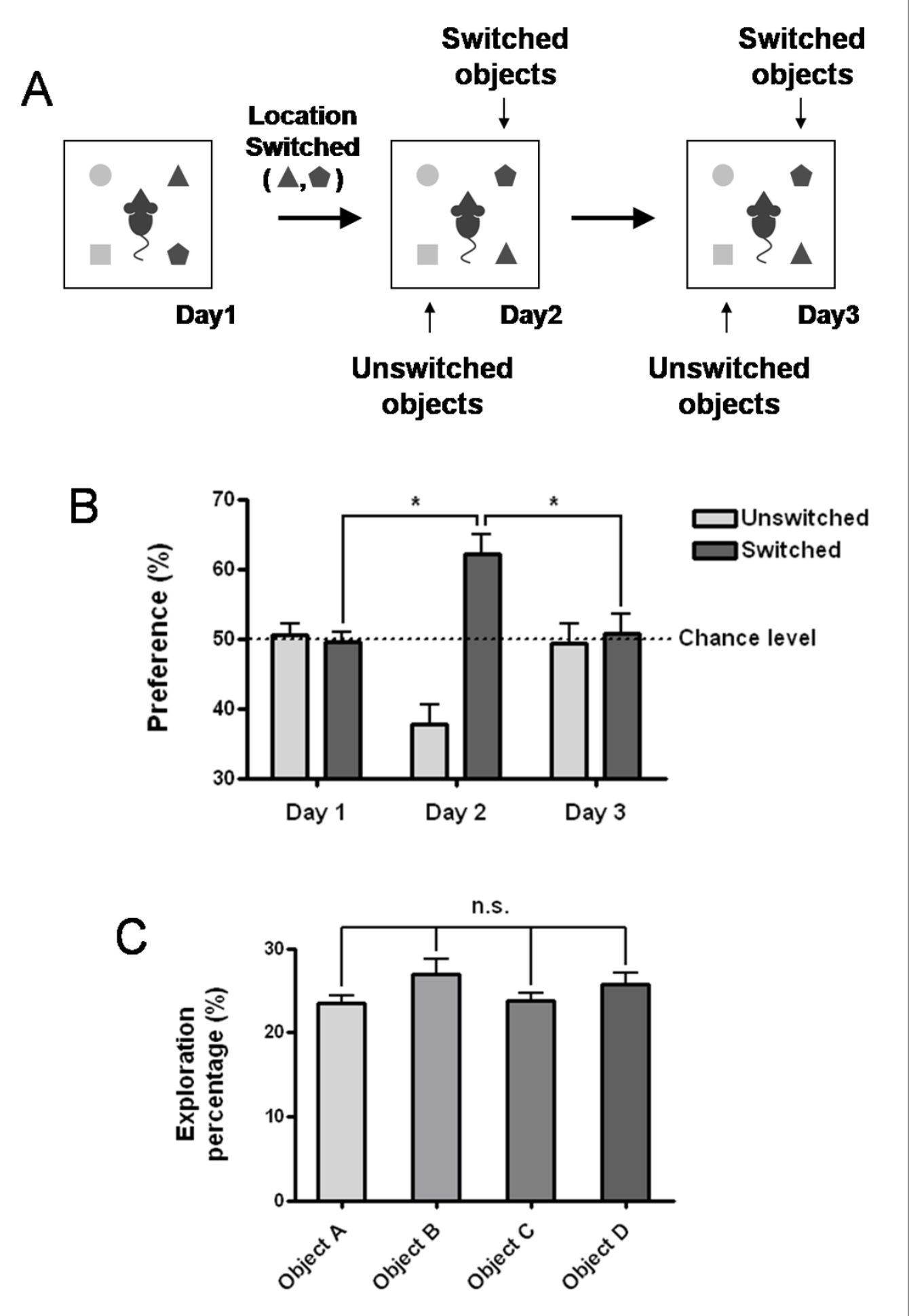

Figure 1 Object rearrangement task. A, Schematic view of the task. After five days of habituation to the context, the mice were exposed to four distinctive objects each placed in one of the four positions for 15 minutes (Day 1). Two adjacent objects' positions were switched for the next two days (Day 2, Day 3), exposed for 15 minutes each. The object pair that is switched was counterbalanced between experiments. B, Preference to unswitched and switched objects over 3 days $\left(n=6,{ }^{*} p<0.05\right.$; paired t test). Preference is the percentage of the two switched/ unswitched objects exploration time from the total exploration time. C, Preference to each objects in the first day (n.s., non significant). 


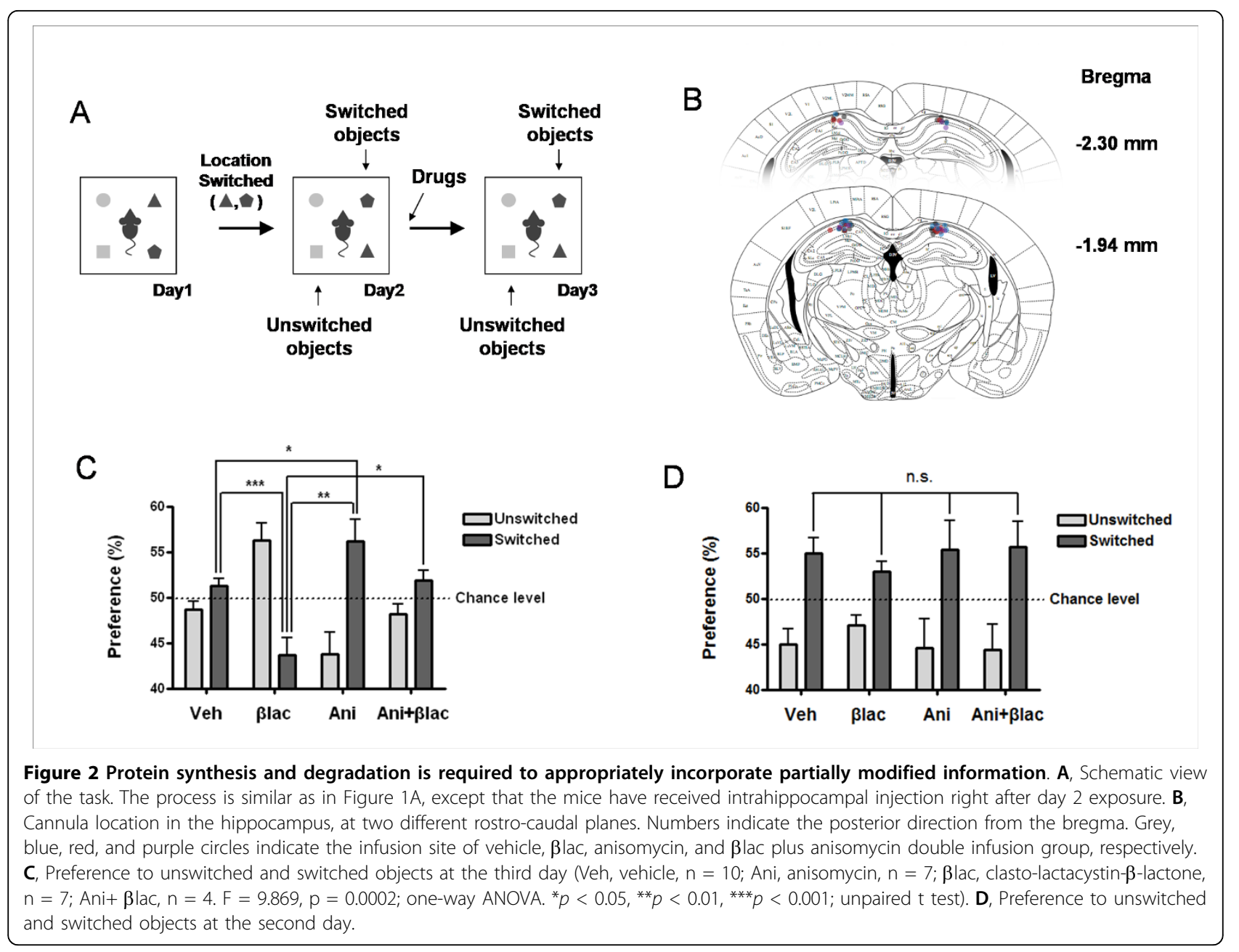

memory becomes labile in this stage to be sensitive to protein synthesis inhibitor. For example, the memories for unswitched objects might have been less affected by anisomycin being maintained relatively intact, while the memories for switched objects may have been more affected, being reactivated and destabilized. This may result in higher explorative preference for the switched objects, as the location for switched objects becomes more novel than the location for the unswitched objects. It is also possible that the consolidation-like process for incorporating additional information (i.e. switched location) is more sensitive to protein synthesis inhibitor than the reconsolidation process. In any case, our result indicates that incorporation of changed information into the pre-existing memory requires de novo protein synthesis.

The interpretation of the effect of proteasome inhibition is more complicated. If the process to incorporate the changed information is a simple additional learning of the novel information, the effect of a certain drug might be expected to be either impairment of modification leaving the memory unchanged or no effect leading to successful incorporation of the new information. In this study, however, the inhibition of protein degradation appeared to affect even the memory for unchanged information. Therefore, memory reactivation on day 2 is likely to have induced complex processes involving modification to the previous memory, in which the protein degradation plays a major role.

Previous reports suggest that destabilization of the reactivated memory precedes the protein synthesisdependent process. However, the retrieval process of four-object rearrangement task differs from that of contextual fear conditioning as it incorporates additional information rather than simply recovers the memory state. Although the memory reactivation-induced protein degradation seems to be critical to form successfully modified memory, the full process may also, in parts, include consolidation-like process to encode the novel memory components. This process may be induced independently of destabilization of the previous memory. In this case, protein degradation inhibition would impair 
destabilization of the reactivated memory and possibly the following restabilization process, without affecting the independent consolidation-like process. The imbalance between these two essential components induced by protein degradation inhibition might have lead to higher preference for the unswitched objects on day 3 . In addition, the protein degradation might have a unique role in either restabilization of the previous memory or in a consolidation-like process to incorporate additional information. We cannot exclude the possibility that protein degradation is involved in the consolidation process in the four-object task used in the present study, although it does not seems to be the case, at least acutely, in contextual fear memory [5].

We have locally treated the inhibitors in hippocampus based on references that indicate hippocampus as a region specifically involved in object location memory rather than the object identity memory. The results using PKMzeta inhibitor demonstrates that hippocampus is at least one of the memory storage sites for object location memory while it is not a critical storage site for object identity memory [18]. Therefore, protein synthesis and degradation inhibition treatment in hippocampus is unlikely to have affected the object identity memory. However, there is a possibility that other brain regions are also critically involved in this memory task.

Although more studies are required to fully understand the mechanism, the present study suggests that protein synthesis and degradation play important roles in modifying the memory in object-location memory paradigm. This evidence supports the hypothesis that memory dynamically changes after retrieval through protein turnover. In addition, we expect that the scheme used here or other modes of the object rearrangement task can be utilized for the investigation into the memory modification mechanism in the future.

\section{Acknowledgements \\ We thank Dr. Yadin Dudai very much for critical comments on the manuscript. This work was supported by National Creative Research Initiative Program of the Korean Ministry of Science and Technology. JHC and JEK are supported by BK21 fellowships. JHC is supported by Seoul Science fellowships. All works were conducted according to the policy and regulation for the care and use of laboratory animals approved by Institutional Animal Care and Use Committee in Seoul National University.}

\footnotetext{
Author details

${ }^{1}$ National Creative Research Initiative Center for Memory, Department of Biological Sciences, College of Natural Sciences, Seoul National University, 151-742 Seoul, Korea. ${ }^{2}$ Department of Brain and Cognitive Sciences, College of Natural Sciences, Seoul National University, 151-742 Seoul, Korea.

\section{Authors' contributions}

JHC: experiment design and implementation, manuscript writing.

JEK: behavior experiments and sample preparation

BKK: experiment design and advice, manuscript writing.

All authors read and approved the final manuscript.
}

\section{Competing interests}

The authors declare that they have no competing interests.

Received: 28 October 2009

Accepted: 8 January 2010 Published: 8 January 2010

\section{References}

1. Suzuki A, Josselyn SA, Frankland PW, Masushige S, Silva AJ, Kida S: Memory reconsolidation and extinction have distinct temporal and biochemical signatures. J Neurosci 2004, 24:4787-4795.

2. Nader K, Schafe GE, Le Doux JE: Fear memories require protein synthesis in the amygdala for reconsolidation after retrieval. Nature 2000, 406:722726.

3. Milekic $\mathrm{MH}$, Alberini $\mathrm{CM}$ : Temporally graded requirement for protein synthesis following memory reactivation. Neuron 2002, 36:521-525.

4. Dudai $Y$ : Reconsolidation: the advantage of being refocused. Curr Opin Neurobiol 2006, 16:174-178.

5. Lee SH, Choi JH, Lee N, Lee HR, Kim Jl, Yu NK, Choi SL, Kim H, Kaang BK: Synaptic protein degradation underlies destabilization of retrieved fear memory. Science 2008, 319:1253-1256.

6. Helton TD, Otsuka T, Lee MC, Mu Y, Ehlers MD: Pruning and loss of excitatory synapses by the parkin ubiquitin ligase. Proc Natl Acad Sci USA 2008, 105:19492-19497.

7. Rodriguez-Ortiz CJ, De la Cruz V, Gutierrez R, Bermudez-Rattoni F: Protein synthesis underlies post-retrieval memory consolidation to a restricted degree only when updated information is obtained. Learn Mem 2005, 12:533-537.

8. Morris RG, Inglis J, Ainge JA, Olverman HJ, Tulloch J, Dudai Y, Kelly PA: Memory reconsolidation: sensitivity of spatial memory to inhibition of protein synthesis in dorsal hippocampus during encoding and retrieval. Neuron 2006, 50:479-489.

9. Rossato Jl, Bevilaqua LR, Myskiw JC, Medina JH, Izquierdo I, Cammarota M: On the role of hippocampal protein synthesis in the consolidation and reconsolidation of object recognition memory. Learn Mem 2007, 14:36-46.

10. Rodriguez-Ortiz CJ, Garcia-DeLaTorre P, Benavidez E, Ballesteros MA, Bermudez-Rattoni F: Intrahippocampal anisomycin infusions disrupt previously consolidated spatial memory only when memory is updated. Neurobiol Learn Mem 2008, 89:352-359.

11. Dix SL, Aggleton JP: Extending the spontaneous preference test of recognition: evidence of object-location and object-context recognition. Behav Brain Res 1999, 99:191-200.

12. Ko HG, Jang DJ, Son J, Kwak C, Choi JH, Ji YH, Lee YS, Son H, Kaang BK: Effect of ablated hippocampal neurogenesis on the formation and extinction of contextual fear memory. Mol Brain 2009, 2:1.

13. Jenkins TA, Amin E, Pearce JM, Brown MW, Aggleton JP: Novel spatial arrangements of familiar visual stimuli promote activity in the rat hippocampal formation but not the parahippocampal cortices: a c-fos expression study. Neuroscience 2004, 124:43-52.

14. Bachevalier J, Nemanic S: Memory for spatial location and object-place associations are differently processed by the hippocampal formation, parahippocampal areas TH/TF and perirhinal cortex. Hippocampus 2008, 18:64-80.

15. Lee I, Solivan F: The roles of the medial prefrontal cortex and hippocampus in a spatial paired-association task. Learn Mem 2008, 15:357-367.

16. Gilbert PE, Kesner RP: Memory for objects and their locations: the role of the hippocampus in retention of object-place associations. Neurobio/ Learn Mem 2004, 81:39-45.

17. Gilbert PE, Kesner RP: Role of the rodent hippocampus in pairedassociate learning involving associations between a stimulus and a spatial location. Behav Neurosci 2002, 116:63-71.

18. Hardt $\mathrm{O}$, Migues PV, Hastings M, Wong J, Nader K: PKMzeta maintains 1day- and 6-day-old long-term object location but not object identity memory in dorsal hippocampus. Hippocampus 2009.

doi:10.1186/1756-6606-3-1

Cite this article as: Choi et al:: Protein synthesis and degradation are required for the incorporation of modified information into the preexisting object-location memory. Molecular Brain 2010 3:1. 